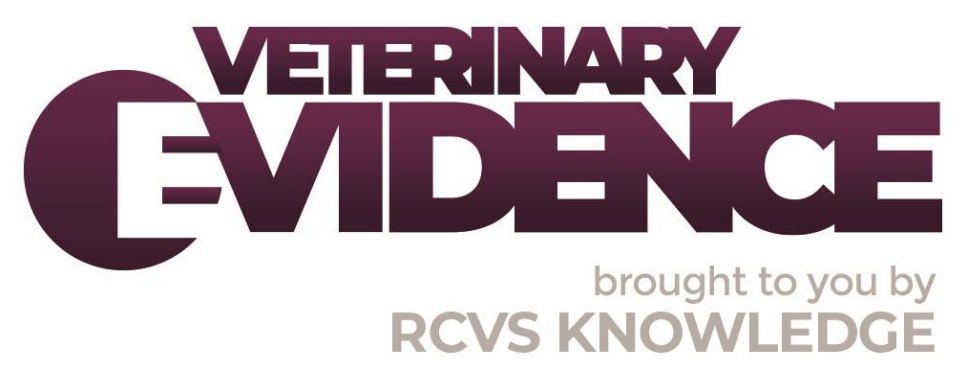

\title{
Veterinary Telemedicine: A literature review
}

Lori Massin Teller DVM DABVP (canine/feline) CVJ ${ }^{1}$

Heather K. Moberly MSLS AHIP FHEA PgCert (Vet Ed) ${ }^{1,2^{*}}$

${ }^{1}$ College of Veterinary Medicine and Biosciences, Texas A\&M University, College Station TX 77843-
4461 USA
${ }^{2}$ University Libraries, Texas A\&M University, College Station, TX 77843-4462 USA
${ }^{*}$ Corresponding Author ( $\underline{\text { hmoberly@library.tamu.edu) }}$

ISSN: 2396-9776

Published: 30 Oct 2020

in: The Veterinary Evidence journal Vol 5, Issue 4

DOI: 10.18849/VE.V5I4.349

Reviewed by: Adam Swallow (BVSc MRCVS) and Collette Taylor (BVSc MRCVS) 
As telemedicine becomes more mainstream in the veterinary profession, it is important to understand when and how to utilise it successfully, and its potential downsides. This literature review supports the use of veterinary telemedicine for teleconsultations, and using wearable and mobile health (mHealth) devices for monitoring animal health. Data supporting the provision of virtual care directly to a client within an established veterinarian-client-patient relationship (VCPR) is more limited, and some of what we know comes from paediatric medicine on the human side. As we have learned from human health care providers, we must be aware there could be a tendency to overprescribe antimicrobials in a virtual visit compared to an in-person visit. Data have also shown telemedicine can be just as effective in diagnosing respiratory disease when compared to traditional visits to a doctor's office or hospital. Telemedicine is especially effective in areas where access to care is limited, whether because of geography, finances, or lack of resources. Overall, veterinary telemedicine and telehealth can provide positive results.

\section{INTRODUCTION}

Telemedicine, telehealth, and virtual care are recent buzzwords in veterinary medicine, despite veterinarians practicing it for years. The Animal Medical Center and Dr. Tilley (DVM) are credited with introducing telemedicine through telecardiology in 1980 by transmitting electrocardiograms over telephone lines for interpretation by a cardiologist or internist thus allowing primary care providers to benefit from advice from experts thousands of miles away (Robertson, 1999). Virtual care is an extension of veterinary practice that includes any interaction among clients, patients, and their circle of care that occurs remotely, using any form of technology, with the goal of delivering quality and effective patient care (American Veterinary Medical Association \& American Animal Hospital Association, 2018). It encompasses telehealth, telemedicine, and mobile health (mHealth). This narrative review explores the literature of veterinary virtual care through four lenses: the overarching context of telemedicine in human health, teleconsulting (veterinarian-to-veterinarian), telemedicine (veterinarian-to-client), and $\mathrm{mHealth} /$ wearables/smartphone applications (apps).

Telemedicine is not a single or standardised method and as such, a review about it must begin with an overview of the scopes and definitions. This paper focuses on practice in the United States, United Kingdom, and Western Europe and employs definitions of the topic from leading organisations in these locales.

The American Veterinary Medical Association (AVMA) defines telehealth as 'the overarching term that encompasses all uses of technology geared to remotely deliver health information or education'. It defines telemedicine as the use of medical information exchanged from one site to another via electronic communications to improve a patient's clinical health status'. It also defines mobile health or mHealth as 'a subcategory of telehealth that employs mobile devices,' including wearables and mobile applications (AVMA Practice Advisory Panel, 2017).

The Royal College of Veterinary Surgeons (RCVS) (2018) states 'telemedicine is the use of electronic communication and information technologies to provide clinical healthcare remotely'. It further states 'telemedicine extends to the provision of veterinary services by video link, text, instant messaging or telephone, or by any other remote means,' (RCVS, 2018). 
Another definition to understand is the veterinarian-client-patient relationship (VCPR). The AVMA (2019) defines it as:

The veterinarian-client-patient relationship is the basis for veterinary care. To establish such a relationship the following conditions must be satisfied:

1. The licensed veterinarian has assumed the responsibility for making medical judgments regarding the health of the patient(s) and the need for medical therapy and has instructed the client on a course of therapy appropriate to the circumstance;

2. There is sufficient knowledge of the patient(s) by the veterinarian to initiate at least a general or preliminary diagnosis of the medical condition(s) of the patient(s);

3. The client has agreed to follow the licensed veterinarian's recommendations;

4. The licensed veterinarian is readily available for follow-up evaluation or has arranged for:

1. Emergency or urgent care coverage, or;

2. Continuing care and treatment has been designated by the veterinarian with the prior relationship to a licensed veterinarian who has access to the patient's medical records and/or who can provide reasonable and appropriate medical care.

5. The veterinarian provides oversight of treatment;

6. Such a relationship can exist only when the veterinarian has performed a timely physical examination of the patient(s) or is personally acquainted with the keeping and care of the patient(s) by virtue of medically appropriate and timely visits to the operation where the patient(s) is(are) kept, or both;

7. Patient records are maintained.

The RCVS does not have a written definition of VCPR, however they provide information in their Code of Professional Conduct (RCVS, n.d. [Accessed 6 October 2020]):

2.29 Specific advice provided remotely, for example via phone or video-link with or without additional physiological data (commonly referred to as telemedicine or telehealth), should only be given to the extent appropriate without a physical examination of the animal. The more specific the advice, the more likely it is that the animal's owner should be advised to consult a veterinary surgeon in person for a physical examination. In this scenario, the animal owner should be asked to provide the veterinary surgeon carrying out the physical examination with a copy of any advice given remotely.

2.30 Veterinary surgeons should ensure as far as possible that the provision of specific advice provided remotely does not compromise welfare, since the animal has not been examined and there is no ability to monitor the animal.

The Federation of Veterinarians of Europe (FVE) does not have a specific definition of telemedicine or of the VCPR. They do recommend in the European Veterinary Code of Conduct with regards to the utilisation of digital technology (Federation of Veterinarians of Europe, 2019):

'Veterinarians should utilise digital and emerging technologies to enhance their provision of services as long as they can use these technologies competently, and hold up-to-date knowledge of the animal(s), of the owner and/or of the farm(s)/farmer(s).' 
The FVE further believes that an examination is necessary to make a diagnosis and issue a prescription. However, they state that a diagnosis can be made with a physical exam or an investigative examination of samples:

'FVE strongly believes that examination, diagnosis, recommendations for subsequent action, and the prescription of medicines or surgery are all strongly linked and must be the exclusive preserve of the veterinarian. A diagnosis cannot be made without examination, either physical examination of the animal or investigative examination of samples. A treatment cannot be recommended or a surgical intervention performed without an examination and a diagnosis. Neither can a veterinary prescription be issued without a diagnosis.' (Federation of Veterinarians of Europe, 2019)

Despite the recent surge in popularity of veterinary telemedicine, few narrative reviews were identified (Devi et al., 2015; and Mars \& Auer, 2006). Veterinary telemedicine is not a new topic, commentaries, opinions, and case reports have been published since the 1980s (Araújo et al., 1999; Robertson, 1999; and Tilley et al., 1986). In 2001, Papageorges et al. wrote several articles about telemedicine ranging from an overview of ' $w$ hat it is' to 'how to make it work' and 'how to choose a system to use,' (Papageorges, 2001; Papageorges et al., 2001; and Papageorges \& Hebert, 2001a). These were part of a 2001 theme issue in Clinical Techniques in Small Animal Medicine about veterinary telemedicine (Papageorges, 2001). In 2007, the Italian Society of Veterinary Science (SISVet) [sic] recognised the potential impact telemedicine could have on the veterinary profession and created the first veterinary telemedicine study group. They brought together veterinarians, medical researchers, engineers, physicians, and lawyers with the goal of contributing to the development of veterinary telemedicine in Italy and the European Union (Forlani et al., 2010). More recently some evidence-based articles documented the role of telemedicine in the veterinary profession. This is discussed later in the paper.

\section{METHODS \& MATERIALS}

The objective of this narrative review is to survey the literature of veterinary telehealth. The search report models the PRISMA-S Draft Reporting Guidelines to the best ability of the researchers (Rethlefsen et al., 2019). A veterinary information specialist/librarian (Moberly) in consultation with a veterinary telemedicine expert (Teller) constructed and executed all searches.

\section{Information sources and methods}

A comprehensive literature search was conducted using three databases, citation searching, hand searching, and alerts. The search strategy was peer-reviewed. Initial searches were conducted on 10 May 2019. They were peer-reviewed, updated several times during the project, and conducted for the final time on 21 January 2020.

Searches were conducted in PubMed using the Legacy algorithm, CAB Abstracts using the OVID search platform, and ProQuest Dissertations \& Theses Global Abstracts using the ProQuest search platform (CAB Abstracts, n.d.; National Library of Medicine et al., n.d.; and Palmer, n.d.). Citation searching consisted of browsing reference lists and following citing references to both keystone articles and those presenting underrepresented data. Citing references were followed using Web of Science and Google Scholar. No searches were conducted simultaneously on a single platform and no study registries were searched. No additional studies or data were sought; however, one author was contacted and asked for clarification of an article. Online browsing of four publications (Clinician's Brief, Veterinary Team Brief, DVM 360, and Today's Veterinary Business) was conducted at each publication website using the native search interface (Today's Veterinary Business | An Official Journal of the NAVC, n.d.). These publications were tallied in the hand searching count. 


\section{Search strategies}

The final search strategies are presented below in a format designed to be copied and pasted for replication. Screenshots of the searches within the database and search platform are available from the authors upon request.

PubMed Search via Legacy platform and algorithm:

( ( ( ( ( ( telecommunication OR telecommunications OR telecommunicat* OR teleconsult OR teleconsultation OR teleconsultations $O R$ teleconsult* $O R$ "virtual visit" OR "virtual visits" OR "virtual care" OR telehealth OR telehealth* OR telemedicine OR telemedicin* OR videoconference OR videoconferences OR videoconferencing OR videoconfer* OR "mobile phone" OR "mobile phones" OR smartphone OR smartphones OR telephone[MeSH] OR "cell phone" OR "cell phones" OR "remote consult" OR "remote consultation" OR "remote consultations" OR teleradiology OR telepathology OR telepatholog* OR telediagnosis OR telediagnos* OR telepet OR petfax OR telemetry)))))) AND (((("Practice Management, Veterinary"[Mesh]) OR (((Office Management[MeSH] AND Veterinary Medicine[MeSH]))))) OR $((((($ veterinary medicine[MeSH Terms]) OR veterinary[Title/Abstract] )))))

\section{CAB Abstracts via OVID:}

((telecommunication or telecommunications or telecommunicat* or teleconsult or teleconsultation or teleconsultations or teleconsult* or "virtual visit" or "virtual visits" or "virtual care" or telehealth or telehealth* or telemedicine or telemedicin* or videoconference or videoconferences or videoconferencing or videoconfer* or "mobile phone" or "mobile phones" or smartphone or smartphones or "cell phone" or "cell phones" or "remote consult" or "remote consultation" or "remote consultations" or teleradiology or telepathology or telepatholog* or telediagnosis or telediagnos* or telepet or petfax or telemetry).mp. or (mobile telephones/ or telecommunications/ or telemedicine/)) AND ((veterinarian or veterinarians or veterinary).mp. or (veterinary medicine/ or veterinary science/ or veterinary services/ or veterinarians/))

ProQuest Dissertations \& Theses Global via ProQuest platform:

noft(veterinar*) AND noft(telecommunication OR telecommunications OR telecommunicat* OR teleconsult OR teleconsultation OR teleconsultations OR teleconsult* OR "virtual visit" OR "virtual visits" OR "virtual care" OR telehealth OR telehealth* OR telemedicine OR telemedicin* OR videoconference OR videoconferences OR videoconferencing OR videoconfer* OR "mobile phone" OR "mobile phones" OR smartphone OR smartphones OR "remote consult" OR "remote consultation" OR "remote consultations" OR teleradiology OR telepathology OR telepatholog* OR telediagnosis OR telediagnos* OR telepet OR petfax OR telemetry)

Searches were conducted with no limits. No limits were applied to results. No published search filters were used and no search strategies from previously published literature reviews were adapted or reused. Searches in PubMed, CAB Abstracts and ProQuest Dissertations \& Theses Global were updated by rerunning searches on several occasions. A comprehensive literature search was conducted using three databases, citation searching, hand searching, and email alerts of new results from database searches. Final searches were conducted in PubMed, CAB Abstracts, and ProQuest Dissertations \& Theses Global on 21 January 2020.

Online browsing of four publications DVM 306, Clinician's Brief, Veterinary Team Brief, and Today's Veterinary Business was conducted at each publication website. These search interfaces were relatively unsophisticated. They each allowed truncation and returned results in a relevancy ranking. Terms searched were teleconsult*, telemed*, telecommun*, telepath*, telediagn*, telerad*, telepet, and petfax. Clinician's Brief and Veterinary Team Brief use the same platform, however, they need to be searched separately. The site allowed truncation and content selection. For each publication, the Articles section was searched. Results were presented in a ranked order and up to the first 50 for each term were evaluated. Today's Veterinary Business uses a custom Google search for the website which allowed truncation. The DVM 360 site simultaneously searched two other publications, Vetted Magazine and Firstline Magazine, and results from there were disregarded. DVM 360 site 
allowed searching of 2018 and 2019 when searched in 2019. It allowed only 2019 and 2020 when searched in 2020. Searches of these publications were last updated 21 January 2020.

\section{Peer-review}

Initial search strategies were peer-reviewed by Clare Boulton, Head of Library and Knowledge Services, Royal College of Veterinary Surgeons Knowledge. Search strategies were edited and finalised based on peer-review input.

\section{Managing records}

A total of 191 records were identified in PubMed, 249 records in CAB Abstracts, and 17 in ProQuest Dissertations \& Theses Global. Additionally, 42 records were identified through hand searching. This resulted in 306 records after deduplication.

Database results were exported from the databases into Zotero, a cloud-based citation management system, (Corporation for Digital Scholarship, n.d.). An effort was made to locate each record identified through hand searching in a database and exporting to add to Zotero in order to keep the information collected about each record consistent. Duplicates were identified using the Zotero Duplicate Items function and individually verified before removal. Five articles (10 items) were duplicates and treated as such; however, they did legitimately have different citations because they were identical articles each included in two tracks of the same conference (a veterinarian track and a veterinary technician/nurse track).

\section{Inclusion criteria}

Inclusion criteria included an English language version of the article being available. There were no date or geographical limits. The topic is veterinary telemedicine, defined broadly, including remote monitoring of health characteristics and evaluation of client acceptance where it related to telemedicine.

\section{Exclusion criteria}

Exclusion criteria included articles where no English language version of the article could be located. Topical exclusions included aquaculture, telephone technology from the 1970s, remote monitoring of the physical location of animals, technology related to education, teaching distance education, client experience, telecommunication skills, marketing, conservation, infrastructure disparities (e.g. broadband), information technology, telecommunications, veterinary smartphone applications not related to telemedicine (e.g. drug dose calculations).

\section{RESULTS}

\section{Telehealth in human health}

Telehealth is a topic of significant discussion in human health care, generating a large literature that includes review articles; specialised guidelines and applications; and summaries about utilisation, reliability, and prescribing of antimicrobials (Burke et al., 2015; Kane \& Gillis, 2018; McSwain et al., 2017; Ray et al., 2019; Shigekawa et al., 2018; and Siew et al., 2016). Kane and Gillis (2018) analysed physician use of telemedicine and found in 2016 that $15 \%$ used telemedicine for patient interactions and $11 \%$ used it to interact with other physicians. This was especially true of physicians in non-metropolitan areas. Physicians in larger practices were also more likely to use telemedicine than those in smaller practices. This is most likely due to the financial burden of implementation (Kane \& Gillis, 2018). Shigekawa et al. (2018) published a rapid review of systematic 
reviews and meta-analyses on patients' use of telemedicine. They set out to answer two questions. First, does the research evidence indicate if telehealth services are equivalent to in-person services? Second, does it indicate whether the use of telehealth services affects the use of other services? The research indicated that in most cases, telehealth was equivalent to in-person care, and in some areas, like telerehabilitation and telenutrition, it was better. Results varied for teledermatology, which revealed live video visits were equivalent, but store-and-forward, or asynchronous, care was not. Few reviews covered the effects of telehealth's impacts on other healthcare services, so the authors could not reach a conclusion in that regard. They did conclude telehealth has the potential to improve access to care for people in rural communities, those with transportation barriers, and those facing provider shortages (Shigekawa et al., 2018).

When veterinary telehealth discussions arise, frequent comparisons are made to paediatrics, especially to the care of infants and toddlers because neither they nor animals can reliably communicate their feelings or complaint. In 2017, the American Telemedicine Association (ATA), founded in 1993, created a set of guidelines and operating procedures for the use of telehealth in paediatric medicine. These have been endorsed by the American Academy of Pediatrics (AAP) (McSwain et al., 2017). This endorsement 'automatically expire(s) 5 years after publication unless reaffirmed, revised, or retired at or before that time,' (American Academy of Pediatrics, 2017). Burke \& Hall (2015) state:

Teleconsultation works well for both acute and chronic disease. Advantages of such consultations include increased access for the medically underserved, improved access for the rural and inner-city child, enhanced care through faster and more accurate assessment than can be provided by telephone consultation, and decreased cost to the health care system and the patient's family. Telepractice involves establishing links between doctors and their patients who may be located in a child care centre, preschool, school, or juvenile detention facility. Telepractice does not replace the in-person visit but rather adds to it. The advantages of such links include an enhanced medical home in which personal physicians care for children; reduced health care system costs, as well as fewer school absences for the children; less money spent by parents on travel; less time away from employment for parents; and less crowding in emergency departments.

Interestingly, veterinarians often quote a statement in the paediatric telehealth guidelines:

Telehealth services should not be provided to children under two years of age in their home or other non-clinical setting except when the provider or their surrogate primary care medical home $\left(\mathrm{PCMH}^{*}\right)$ has referred them for subspecialty consultation (McSwain et al., 2017).

*PCMH - Patient-Centered Medical Home

Some interpret this to mean that using telehealth is not an acceptable modality of care for infants and toddlers, so it must not be acceptable for animals either. When McSwain was contacted by this author and AVMA staff for clarification on that statement, he provided a written letter to disabuse that notion. His letter (McSwain, personal communication, 2019) states:

The above language was provided out of the recognition that children below the age of 2 years represent a high-risk population with significantly different care management best practices for common conditions than for older children. As an example, a urinary tract infection requires significantly more diagnostic work up for a child in this age group than for an older child due to the risk of an underlying causative anatomic abnormality. As such, continuity of care with a medical home was considered important by the authors as often the management cannot be completed in one encounter. Additionally, examination via telemedicine may be complicated by the lack of tools that are appropriately sized or validated in this age range. 
The ability of the patient to communicate with the provider was not a significant consideration in the development of this provision. In most cases of pediatric telehealth, there is a guardian present during the encounter. Conversely, telehealth is a valuable tool in many situations and populations in which the patient is non-communicative for a variety of reasons, including behavioral, developmental, and other medical conditions. Access to care afforded by telehealth is particularly important for high risk populations. The ability to intervene early and leverage the continuum of care afforded by telehealth are benefits which we would encourage all healthcare fields to consider.

Siew et al. (2016) assessed the reliability of telemedicine in seriously ill children. They examined 132 febrile children between the ages of 2 and 36 months and 145 children between the ages of 2 months and 18 years with respiratory distress. For all cases, they found good to excellent agreement between the telemedicine and bedside exams, and they concluded telemedicine is reliable in the assessment of febrile children and those with respiratory distress (Siew et al., 2016).

A lesson veterinarians can learn from paediatric virtual care visits is to be cautious about prescribing antibiotics. Ray et al. (2019) published a study comparing prescribing antibiotics for acute respiratory infections in children 0-17 years of age. They looked at the number of prescriptions written for direct-toconsumer (DTC) telemedicine visits as compared to urgent care visits and with visits to the primary care provider. Children who had co-morbidities were excluded in this study. The study indicated antibiotic prescribing was highest in DTC telemedicine visits with $52 \%$ of patients receiving a prescription, compared to $42 \%$ of urgent care visits and $31 \%$ of primary care visits (Ray et al., 2019).

\section{Teleconsulting (veterinarian-to-veterinarian)}

Teleradiology, a subset of teleconsulting that began in the mid-1980s, allows an attending veterinarian to take images, such as radiographs, CTs, or MRIs and send them to a radiologist for interpretation. The radiologist provides a report with their findings, interpretation, potential differential diagnoses, other tests to consider, and/or possible treatment options (Poteet, 2008; and Tilley et al., 1986). Veterinarians paid a fee for these services and passed them to the client as an accepted part of patient care. In 2001, nearly two decades ago, Papageorges \& Tilley (2001) asked, 'Why telemedicine?' One example was a veterinarian outside of Houston, Texas, who started to send all his radiographs, sonograms, and cytology to specialists via a telemedicine connection. Telemedicine allowed him to elevate his practice from vaccines and a "shotgun" approach to complicated problems and provided the ability to practice higher quality medicine in a more efficient and accurate manner. His practice became more profitable as a result (Papageorges \& Tilley, 2001). Martinelli (2001) also noted that the large ratio of general practitioners to specialists provided 'ample impetus for a telemedicine system' and telemedicine had advantages compared to a telephone consult, including the ability to review pertinent documents and images.

The Job Analysis of the American College of Veterinary Radiology regularly surveys radiologists asking about the procedures they perform, species they see, and how they spend their time to better tailor the objectives of radiology residency training (Fischetti et al., 2017). In the most recent analysis, 37\% of the diplomates engage in teleradiology and there is a significant trend of radiologists moving from academia to private practice. To help ensure there are enough radiologists to meet the needs of the profession, hybrid residencies bridge private practice and the academe. These private practices contribute partial funding and the academic institutions provide core educational objectives not available in a private practice, like large animal imaging or nuclear medicine. There is no obligation for the resident to remain with the private practice or academic institution at the completion of the residency. This contrasts with corporate residency sponsorship with an obligation for the resident to immediately work for the corporate sponsor and practice teleradiology 
preventing the newly boarded radiologist from the opportunity to develop and mature in a veterinary hospital setting where there is the opportunity to work with colleagues and continue to build experience (Fischetti et al., 2017). Noel et al. (2016) compared the accuracy of off-site use of smartphones and a standard computer workstation to diagnose mechanical obstructions in the small intestine of dogs and cats. There were no statistical differences in accuracy based on the device used. The only accuracy differences appeared to come from the years of experience the radiologist had, with the more experienced radiologist obtaining a higher degree of accuracy (Noel et al., 2016).

Papageorges et al. (1998) wrote a letter to the editor of JAVMA stating that telemedicine could alleviate the shortage of veterinarians qualified to interpret sonograms. They pointed out that there are two parts to a diagnostic scan; the skill of the sonographer that collects the images and the abilities of the person reading the scan. At the time that letter was written, there were not enough qualified individuals to interpret sonograms, so one of the solutions to the undersupply was the recommendation to utilise telemedicine (Papageorges et al., 1998). Marr (2001) suggested that telemedicine could be used to improve the sensitivity and specificity of equine ultrasonography techniques. Navas de Solis et al. (2019) documented that real time telehealth using ultrasonography is feasible and can have positive results in equine practices. There were two different phases to this study. In Phase 1, an equine veterinarian in private practice performed an ultrasound exam while connected virtually with a veterinary sonographer at a veterinary college. The sonographer could visualise the ultrasound in real time and provide guidance to the private practitioner. In Phase 2, horses in a teaching hospital had two ultrasound scans. The first exam was done by an inexperienced sonographer (usually a resident) with virtual guidance by a remote expert. This was immediately followed by a scan performed by an expert sonographer who was on-site. In Phase 1, 100\% of the remote interactions were considered useful, for the following reasons: improved case management, increased reassurance to the practitioner and/or owner about the clinical plan, educational value, and rapid input from a specialist without the need to travel. In Phase 2 the residents felt that the remote assistance had both clinical and educational value. In both phases of the study, a drawback was variable internet quality, however, only one consultation had to be discontinued due to poor internet connectivity. The other negative finding, which had been expected, was that the sonograms take longer when performed by inexperienced veterinarians (Navas de Solis et al., 2019).

Other forms of teleconsulting are becoming more common. Digital pathology, also known as virtual microscopy, is increasing with the development of rapid and dependable slide scanners. Whole-slide images can be viewed and manipulated in all directions, and the quality of the image has nearly the equivalent optical resolution and visual magnification of a light microscope (Bertram \& Klopfleisch, 2017). Recommendations for the use of telecytology, especially for both consultations between colleagues and client education, date back to 2001 (Hebert et al., 2001; and Papageorges \& Hebert, 2001b). Maiolino et al. (2006) documented 100\% agreement between a pathologist's telediagnosis and conventional glass slide diagnosis. As digital pathology becomes more common, pathologists can share images with colleagues to obtain a second opinion on a cytology or tissue sample. In this era of big data, automated image analysis is also being studied. Some uses are validated for human pathology, such as certain breast cancers, but work is still needed on the veterinary side (Bertram \& Klopfleisch, 2017).

There are only 86 board-certified veterinary behaviourists in the world (American College of Veterinary Behaviorists, n.d.). Because of the limited numbers, it can be impossible for some owners to obtain an inperson consultation to get help for their animal. The Cummings School of Veterinary Medicine at Tufts University began offering behavioural consults via fax almost 20 years ago. Studies were performed to determine if there was a difference in remote vs. in-person consultations for the treatment of canine aggression toward owners and for the treatment of canine separation anxiety (Cottam et al., 2008; and Dodman et al., 2005). The studies revealed significant improvement in the treatment of both owner-directed aggression and separation anxiety and further showed there was no significant difference in the results whether the consult was handled remotely or in-person. More recently the University of Pennsylvania School 
of Veterinary Medicine is piloting a web-based app to provide behavioural consultations to veterinarians with behaviourists (Carrozza, 2018).

While radiology, pathology, and behaviour may seem like obvious choices for teleconsulting, specialty fields are also utilising virtual care. Papageorges \& Hebert (2001b) suggested many other applications for telemedicine, including cardiology, endoscopy, dermatology, and ophthalmology. Oncologists are teleconsulting support to primary care practitioners because it can be difficult for non-oncologists to keep up with the various treatment modalities for cancer in multiple species. Additionally, clients may not have the resources to travel to a specialist for regular chemotherapy appointments, but a veterinary oncologist can provide support and guidance to an interested general practitioner to appropriately manage the case. Penzo \& Pietersma (2012) outline potential benefits of oncology teleconsultations including: improved access to specialist oncology care, specific case-oriented advice, elevated level of care in a primary care practice, increased profitability from more advanced diagnostics and therapies, and increased range of services offered by the practice. Further benefits include assistance with interpretation of lab work and staging of the cancer, possible prognosis depending on treatment plan that is chosen, palliative care, appropriate drug handling and administration, and educational information for staff and clients. Potential drawbacks may be the inability of the specialist to perform a complete work-up, including physical exam, the skillset of the general practitioner, a perceived threat to the role of the specialist, and concerns about medical liability and malpractice (Penzo \& Pietersma, 2012).

Lanevschi-Pietersma et al. (2011) consider telemedicine both a time management and educational tool for veterinarians and their clients. Virtual consults with a specialist can provide the primary care practitioner a patient-specific written report with recommendations for diagnostics and therapeutics. These can be added to the patient's medical record and a copy provided to the client. There is a difference between a request for a structured consult and an informal consultation that may take place on an electronic forum. Virtual consults also tend to be more efficient than telephone or email exchanges where the appropriate information may not be provided at the time of the request, resulting in much back-and-forth communication. When a primary care practitioner works with a specialist via a virtual visit, clients seem to appreciate the team approach to the patient's care, especially when referral is not an option (Lanevschi-Pietersma et al., 2011).

Niessen \& Forcada (2019) note primary care practitioners appreciate the ability to informally request help from a local specialist or an academician, but the time demands and the sheer increase in demand for these services is leading to the need for scheduled virtual consults occurring in real time. Virtual consults can also provide a support system for veterinarians in primary care practices, especially solo practitioners or those presented with complicated or unusual cases. Often, a specialist or more seasoned practitioner can guide an attending veterinarian in remote ultrasound, ophthalmologic exam, or in the identification of abnormal heart sounds. Digital stethoscopes, otoscopes, ophthalmoscopes, and other technology make some aspects of a virtual exam easier to perform. Veterinarian-to-veterinarian teleconsulting also provides the possibility for mentorship, especially for veterinarians in rural practice or in the case where a younger veterinarian is also a new business owner and is feeling the dual stresses of practice and owning a new business (Niessen \& Forcada, 2019). In an interview in Beef Magazine, Dr. Arn Anderson (DVM) praises the ability of telemedicine, 'Between DVMs, this technology allows us to mentor younger veterinarians in rural environments and allows us to share cases and get second opinions easier and faster. It is better medicine,' (Ishmael, 2015). Dr. Anderson goes on to state rural veterinarians can increase the 'scope and sophistication' of their practice and clients do not have a problem paying for telemedicine services (Ishmael, 2015).

\section{Telemedicine and its role in global health}

Telemedicine is seen as part of the future of animal health care in developing countries where there may be neither enough veterinarians to meet workforce needs nor an adequate veterinary infrastructure. The 
National Research Council (2013) states the veterinary profession needs to expand its capacity to address complex global problems. In India, there is enough work to support 67,000 veterinarians, but there are only 34,500 veterinarians available to meet their needs for both animal and public health (Devi et al., 2015). Animal health care workers in India see the potential for consulting with specialists, including dermatologists, radiologists, pathologists, surgeons, and cardiologists to receive assistance with diagnostic, prognostic, and therapeutic planning. They also appreciate the role remote monitoring can play to improve herd health management and disease prevention. The major barriers to implementing telemedicine in developing countries are interstate licensing issues, confidentiality concerns, broadband and internet connectivity, and potential ethical issues and cost effectiveness (Devi et al., 2015). In developing countries in Africa, telemedicine conducted with smartphone-based technology has been used to increase referrals from paraprofessionals to veterinarians in countries like Kenya and Uganda (Ilukor et al., 2014). Many countries rely on paraprofessionals, defined by the World Organisation for Animal Health (OIE) (2019) as:

a person who, for the purposes of the Terrestrial Code, is authorised by the veterinary statutory body to carry out certain designated tasks (dependent upon the category of veterinary paraprofessional) in a territory, and delegated to them under the responsibility and direction of a veterinarian. The tasks for each category of veterinary paraprofessional should be defined by the veterinary statutory body depending on qualifications and training, and in accordance with need.

Because of potentially detrimental impacts on animal disease, public health, and the overuse of antimicrobials by paraprofessionals operating without veterinary oversight, the OIE recommends strengthening the bond between paraprofessionals and veterinarians (Ilukor et al., 2014; and OIE, 2019). With this improved bond, expectations include more appropriate pharmaceutical prescriptions, improved diagnoses, more accurate disease surveillance, and better adherence to veterinary care standards. Ilukor et al. (2014) noted paraprofessionals with smartphone devices were much more likely to refer to a veterinarian because these devices increased access and allowed better communication. Veterinarians also saw clientele growth when they improved the bond with paraprofessionals and encouraged referrals. Increased referrals are expected to lead to decreases in the prevalence of infectious and zoonotic diseases, like brucellosis, tuberculosis, and Rift Valley fever, leading to public health improvements. Some potential impediments are lack of broadband connectivity and the difficulty of farmers to differentiate between the quality of care provided by paraprofessionals vs veterinarians, both of whom are called animal doctors (llukor et al., 2014).

Global health has become increasingly more important to ensure a healthy food supply and in the identification of potentially zoonotic emerging diseases. In addition to the benefits telemedicine can provide to address these issues in developing countries, it can also be used as an educational tool for students to better understand these problems and the role developed countries can play in helping to resolve them (Mazan et al., 2015). In many developing countries, the population remains dependent on small-scale agriculture for income. Working equids, like donkeys, mules, and horses are the mainstay of the agriculture industry. Though people depend on working equids, particularly donkeys, the farmers frequently do not have the financial resources to provide veterinary care. The Cummings School of Veterinary Medicine at Tufts University partnered with the veterinarians at the American Fondouk in Fez, Morocco, and the Equitarian Initiative (Minnesota, U.S.) to provide speciality veterinary care via teleconsulting to the farmers and their equids. In addition, students participated in these virtual visits and learned about problems faced in developing countries. Calling their programme "Patients without Borders" the two institutions and initiative utilised both synchronous video visits and asynchronous exchanges of information. The donkeys benefitted from expert care that the farmers did not otherwise have access to, and the students benefitted from new educational experiences. The biggest obstacles were unstable internet connections and time zone differences. Imaging - whether radiographs, sonograms, or endoscopic exams - were provided ahead of video visits to limit the use of bandwidth during virtual visits and to allow the faculty and students time to review the information. Students saw medical conditions they would not see in the U.S. or conditions they might have 
been familiar with but required different treatment options because of limited resources available in Morocco (Mazan et al., 2015).

\section{Telemedicine (veterinarian-to-client)}

This review identified less peer-reviewed research about assessing virtual care between a veterinarian and the animal-owning client. Many opinions and editorials discuss both the benefits and concerns around providing telemedicine visits to the animal owner, whether a veterinarian-client-patient relationship (VCPR) exists or not. Gyles (2019) noted the value of a telemedicine service is it can guide clients to the 'appropriate level of intervention' and clients are willing to pay for this convenience. The consistent message across many of these opinion pieces and editorials describes the benefits of telemedicine: improved access to care, client convenience, enhanced veterinary-to-client bond, reducing the workload on front office staff, and a better choice than the client consulting the internet (Freiman, 2019; and Lacroix, 2017). In addition, the articles also address concerns, like risks of missed diagnoses, lack of physical exams, differences in communicating via technology compared to in-person, monetisation of virtual care visits, and limitations of technology (Limb, 2018; and Milani, 2009). Many of these pieces also question if there is a need to establish a VCPR virtually or if it must be established with a hands-on physical exam, or a timely visit to the premises where animals are maintained, in every instance (Cary \& Massecar, 2017; and Cushing, 2017). Others accept virtual care is part of veterinary medicine, are ready to incorporate new technologies into their practice, and want regulatory bodies to keep pace (Nelson-Pratt, 2018). Veterinary teams are encouraged to think outside the box and find ways to help their clinicians incorporate virtual care into the practice (Cushing \& Lacroix, 2018; and Rose, 2017). Many veterinarians are interested in using telemedicine in their practices, but they have concerns about further infringement on their personal time or if telemedicine will disrupt their already busy day-to-day workflow (Freiman, 2019).

There are also legal and ethical concerns when it comes to virtual care cases between veterinarians and clients (Flemming, 2003; and Marshall, 2019). Almost every jurisdiction around the world requires a license to practice veterinary medicine and most have some sort of definition in statute that defines the practice of veterinary medicine. Some states in the U.S., like Kentucky, consider the act of providing general advice to be the practice of veterinary medicine, whether there is an established VCPR or not. Others consider general advice to be allowable, but there must be an established VCPR to make a diagnosis, create a treatment plan, or prescribe medications (Flemming, 2003). There are also concerns about the location where the VCPR is established and where the veterinarian is licensed and how that will be enforced. This is important from both a regulatory perspective and a liability perspective (Jack, 1999). It is generally accepted that technology and innovation advance more quickly than regulations and ethical considerations related to them. Some ethical concerns to consider as virtual care and other technologies evolve include data usage, client privacy, and security of medical records and credit card data (Marshall, 2019).

Professional regulatory agencies in Europe have not paid much attention to veterinary telemedicine, and in Portugal it is not considered an acceptable form of practice by the Portuguese Veterinary Order (Ordem dos Médicos Veterinários, OMV) which prohibits virtual examinations and prescriptions, though these restrictions were temporarily lifted during the COVID-19 pandemic (Mãgalhaes-Sant'Ana et al., 2020). As part of its preparations for a new Code of Professional Conduct, the OMV conducted a Policy Delphi Study in 2018 to determine the views of Portuguese veterinarians about telemedicine to guide policymaking. The vast majority of respondents agreed that there should be limits on the use of telemedicine in practice, and they further believed that OMV should encourage digital literacy and certification of telehealth providers. In the comments, 'most participants acknowledged that the service provided by teleconsultations is complementary to that of physical consultations but stressed the need for having a face-to-face interaction before resorting to telematic means. Others questioned this view; a small animal practitioner noted that in urgent cases, such as poisoning and heat stroke, performing a teleconsultation 'can mean the difference between life and death.' It was also 
mentioned, namely by a specialist respondent, that, in the case of behavioral medicine, since examining animals in their home environment is particularly beneficial, remote consultations should be allowed for both first consultations and follow-ups, according to the judgment of the specialist veterinarian.' As this study was conducted before the COVID-19 outbreak, the authors recommend further research to determine if opinions have evolved. They also recommend that more research be done on the impacts of telemedicine on animal welfare and antimicrobial resistance (Mãgalhaes-Sant'Ana et al., 2020).

Kogan et al. (2016) surveyed veterinarians to determine how information and communication technologies are utilised in veterinary practices. Responses from 967 veterinarians showed younger veterinarians were more comfortable with newer technologies, like texting, Skype, and social media; however, they were the least likely to engage in consultations after hours and on weekends. Older veterinarians were more likely to support the use of remote monitoring devices. Many veterinarians felt that communicating with their clients via email or text could be less time-consuming than via phone or in-person if appropriate boundaries could be set. Some scenarios they identified as requiring less time with a virtual visit were post-operative recheck visits, management of diabetic patients, and nutritional counselling. Of note, older responders were more likely to identify several scenarios where virtual visits could be appropriate compared to their younger counterparts (Kogan et al., 2016). In a survey of pet owners, that received 1,031 responses, who had had a dog or cat hospitalised for longer than 4 hours, many expressed the desire for more frequent updates than what they received. Over two-thirds wanted updates every 2-6 hours and over half of the respondents were willing to pay a premium for regular updates. Most updates were relayed via phone, but over $38 \%$ of owners would prefer text updates. It was noted that veterinarians may want to consider using more text updates, when appropriate, as those can be less time-consuming than phone updates. Millennial pet owners have cited chat or texting as the most important veterinary service they want (Kogan et al., 2019). One of the weaknesses of data collected by surveys is that the sample sizes may not adequately represent all cross-sections of the veterinary practitioner or pet-owning populations (Kogan et al., 2019; and Kogan et al., 2016).

Hawk (2018) surveyed Facebook users to determine the perception and use of telemedicine by veterinarians and by owners. The sample size of 176 respondents included only seven veterinarians or veterinary students making that population statistically insignificant; however, pet owners responses indicated their three top concerns when interacting with a veterinary practice are the humane treatment of their pets, the ease of scheduling, and a timely response. The owners also had a positive attitude toward veterinary telemedicine, especially about potential emergencies. This was statistically significantly true for people aged 18-44 years old compared to those who were aged 45-75. The study also showed owners were willing to pay for virtual care visits (Hawk, 2018).

Robben et al. (2016) evaluated the impact on pet owners and intensive care unit (ICU) staff of a virtual pet visit system with live video streaming. When a pet was admitted to the ICU, the clients were offered the ability to view their pet through a TelePet live-streaming service (TPS). The pet owners who utilised the TPS were found to be more satisfied with the care their pets received than the pet clients who did not opt to use the service. The vast majority who took advantage of the TPS felt less anxious about their pet and would be willing to pay for the service. The veterinary technicians working in the ICU also had generally favourable responses to the use of the TPS, though tempered by the negative comments they received from a few dissatisfied clients who were concerned about what they saw on the video footage. There was no way for this study to be blinded or randomised, but overall the results revealed clients had positive experiences with it. Some clients were distressed by the video, so TPS would not be appropriate for everyone (Robben et al., 2016). In addition to noting that consumers are willing to pay for telemedicine, it is also important to note that providing telemedicine does not have to costly. Papageorges \& Hebert (2001c) recognised that telemedicine can be performed using standard technologies and available tools instead of purchasing expensive equipment from vendors. They summarise by stating; 'We should harness the power of the Internet to give us the communication tools and the freedom we need to practice medicine the way we believe is best.' 
One of the most frequently recommended telemedicine techniques for veterinarians is post surgical recheck exams. Bishop et al. (2018) randomised 30 client-owned dogs into two groups for examinations following surgical sterilisation. In one group, the dogs were rechecked virtually (telemedicine group) and the dogs in the other group were rechecked in the veterinary clinic (control group). None of the dogs randomised to the telemedicine group had a surgical complication necessitating a visit to the clinic. The most common problem was a slow internet connection reducing the quality of the audio-visual feed. The rechecks were still able to be completed virtually. There were two minor surgical complications in the control group randomised for the inclinic rechecks. Owners from both groups were satisfied with the recheck visits; however, the owners from the telemedicine group felt their dogs were less stressed or fearful than if they had taken them for the in-clinic recheck. These owners would strongly prefer to utilise telemedicine for similar appointments in the future, supporting the hypothesis owners found telemedicine visits to be a useful and satisfactory alternative to inclinic appointments. The owners of the dogs in the telemedicine group also saved, on average, 50 minutes of travel time to and from the clinic. One of the advantages of telemedicine highlighted in this study was the ability of virtual care to reduce stress and anxiety in animal patients. One limitation to the study is, while the patients were randomised to the control or telemedicine groups, there was no way for the owners or practitioners to be blinded to the modality used for the recheck exams, which could bias responses (Bishop et al., 2018).

Military working dogs (MWD) perform a wide variety of tasks including being deployed with U.S. military forces without access to veterinary care. Donham \& Wickett (2018) documented the usefulness of virtual care when a MWD became ill at a remote outstation and sepsis was suspected. The dog was transferred to a Canadian Role II medical facility where more medical resources were available but not a veterinarian. The staff interacted with the MWD's veterinarian in the U.S., who was able to work with them, obtain diagnostic information, formulate a plan to stabilise the dog, facilitate evacuating the dog to a military base in Germany then to his home station for more care. Interestingly, it was the ability to videoconference that made the difference in this dog's care because verbal descriptions did not adequately convey the severity of the dog's condition and the visual impression did. The case report concluded that in 'austere expeditionary settings where medical specialties are frequently unavailable, and given the spread of Internet technology to rural settings, the use of video calling for virtual consultations has the potential to improve care in the deployed setting,' (Donham \& Wickett, 2018).

Veterinarians with exotic animal practices have debated the benefits and concerns with telemedicine too. They recognise the advantages of teleconsulting and the potential benefit for situations with a recent handson examination of the patient, but they have concerns about establishing a direct relationship with a client and providing care to the patient without a physical exam having taken place. Many companion exotic animal practitioners see virtual care has a place in husbandry, environmental, and nutritional issues (Hess, 2017).

\section{mHealth, wearables, smartphone applications}

The growth of wearables, smartphone applications (apps), and other technology makes it easier than ever to collect data about an animal's activity, mobility, food consumption, and other health data.

Cardiac arrhythmias can be difficult to diagnose in dogs, and frequently require the utilisation of a 24-hour Holter monitor. These are not always readily available, do not support long-term monitoring, and some animals are intolerant of wearing them. Recently several studies show promise for alternatives to the Holter monitor in dogs. Brlo $\AA 3 / 4$ nik \& Avbelj (2015) piloted a wireless body electrocardiogram (ECG) to investigate whether it could surmount the limitations of a Holter monitor; the results showed great potential. The sample size was very small, and a study with a larger population of animals is pending ( $B r l o \AA 3 / 4 n i k ~ \& ~ A v b e l j, 2015)$. Lichtenberger et al. (2018) published a preliminary assessment of a single-lead, 14-day ECG adhesive patch monitor (APM). Again, the sample size was small, and the results were comparable to those obtained with a 
24-hour Holter monitor. They found that the adhesive caused some mild skin irritation and walking, running or playing caused intermittent motion artefact, but they could still obtain analysable data over $93 \%$ of the time that the dogs wore the patch (Lichtenberger et al., 2018).

Smartphone apps may also play a role in what information can be gathered and shared through a virtual visit. Two independent prospective studies compared a smartphone-based ECG with a standard 6-lead ECG in dogs and cats. The ECGs were measured simultaneously with the smartphone app using a single-lead recorder coupled to it while the reference ECG was recorded with a standard 6-lead ECG machine. Kraus et al. (2016) performed a study that included 51 dogs and 27 cats. Three board-certified cardiologists read each ECG; there was $100 \%$ agreement in heart rates between the two methods, and intra-observer agreement for rhythm diagnoses was extremely high. The only time where there was significant disagreement was in the QRS polarity readings in cats. It is possible this occurred because the electrodes for the two devices were not placed in the same spots (Kraus et al., 2016). Vezzosi et al. (2016) performed a prospective, multi-centre study with a similar design and evaluated 166 dogs. $20 \%$ of the dogs in this study were healthy, and the remainder had a variety of morbidities, including heart disease, cancer, and respiratory, gastrointestinal or neurologic disease. This study found the smartphone ECG app had $100 \%$ sensitivity and $97.9 \%$ specificity in the detection of arrhythmias, and all cases of atrial fibrillation were correctly identified. The ECG app was also excellent at identifying ectopic beats and may be useful for screening or monitoring dogs with cardiomyopathies for arrhythmias. One limitation to the smartphone ECG app is mean electrical axis cannot be calculated because it captures a single lead (Vezzosi, et al., 2016). However, the study authors concluded the smartphone ECG app may allow veterinarians to identify and manage patients at cage side and share the results via electronic means with specialists (Kraus et al., 2016). Further studies should be done to determine the diagnostic value of owners using the smartphone app in the home setting and sharing the results with the veterinarian. Vezzosi et al. (2018) also recently validated a smartphone ECG app in a study with 50 horses. The study showed comparable findings to those in dogs, except there was not good agreement on the polarity of $P$ waves. There was excellent agreement for heart rate and rhythm (Vezzosi et al., 2018).

Dogs and cats are not the only animals where monitoring ECGs can be of benefit. Changes in heart rate in cattle can provide warning signs for illness and disease, which require early detection and intervention to prevent spread through a herd. Warren et al. (2008) studied a prototype electrocardiographic pill that would remain in the reticulum and allow for continuous and automatic acquisition of heart rate and core body temperature. The study showed promise in allowing the capture of information about the cattle's health without requiring restraint (Warren et al., 2008).

The Fever Tag ${ }^{\circledR}$ is an ear tag that monitors a calf's body temperature every 15 minutes and alerts when the temperature deviates from normal parameters which may happen up to 72 hours before the calf shows signs of illness, particularly pneumonia. By signalling the onset of illness, a rancher can intervene early in the course of disease, thus limiting morbidity, mortality, and the use of antimicrobials. McCorkell et al. (2014) tested the efficacy of the Fever $\operatorname{Tag}^{\circledR}$ in feedlot calves just obtained from auction and in specific pathogen-free calves experimentally infected with bovine viral diarrhoea virus. There were no instances of false positive diagnoses of bovine respiratory disease (BRD), but there were significant instances of false negatives and inconsistent readings. The study concluded significant improvements were needed in Fever Tag ${ }^{\circledR}$ accuracy and reliability (McCorkell et al., 2014). However, 3 years later, Mahendran et al. (2017) showed that by using the Fever Tag ${ }^{\circledR}$ to monitor calves' body temperature, they could determine when the calves became ill and their response to therapeutic interventions. In addition to sensing body temperature changes from respiratory disease, the Fever Tag ${ }^{\circledR}$ was also useful in diagnosing navel ill and diarrhoea (Mahendran et al., 2017).

Other technology that can aid in the early detection of BRD is a computer-aided lung auscultation system, such as the Whisper ${ }^{\circledast}$ stethoscope. The stethoscope is used to auscultate lung sounds of cattle and then score the sounds from 1-5, with 1 indicating no abnormalities and a low likelihood of BRD. Mang et al. (2015) tested the technology on 561 steers recently arrived at a feedlot. There was very good specificity and sensitivity when 
utilising the device, though there was a wide confidence interval. They further concluded, because of the higher specificity of this device, there were less falsely diagnosed cases of BRD and thus less use of antimicrobials in the feedlot (Mang et al., 2015). Noffsinger et al. (2014) conducted field trials with the Whisper ${ }^{\circledR}$ stethoscope to determine the relative accuracy of Whisper ${ }^{\circledast}$ scores and rectal temperatures in diagnosing BRD. Two data sets were used. The first involved the correlation between rectal temperatures, manual lung scores, and case fatality rates in feedlot cattle pulled for BRD. The second data set evaluated the relative accuracy of the Whisper ${ }^{\circledast}$ scores and rectal temperatures. When taken alone, the Whisper ${ }^{\circledR}$ score was a better predictor of BRD than a rectal temperature $\geq 104.5^{\circ} \mathrm{F}$; however, a Whisper ${ }^{\circledR}$ score of 1 and no fever was the best predictor of survival. Manual lung scores better correlated with case fatality rates. Using the Whisper ${ }^{\circledR}$ score and rectal temperature in combination improved diagnosis and prognosis, and allowed for more judicious use of antimicrobials (Noffsinger et al., 2014).

Cats may easily become stressed with restraint and repeated handling, especially when ill. This can be especially problematic when attempting to acquire a rectal temperature. Quimby et al. (2009) compared digital rectal and microchip transponder thermometry in 40 cats that were experimentally infected with feline herpesvirus type 1 (FHV1). The cats' temperatures were measured both before and after infection with FHV1. There was sufficient agreement between the digital rectal and the microchip transponder thermometry to consider the microchip a valid substitute for obtaining a rectal temperature (Quimby et al., 2009).

Smartphones and accessories are utilised at cage-side in exotic animal practices. These devices are particularly useful when it may be difficult or dangerous to transport wild or zoo animals, or the animal may be in a fragile or debilitated state making transportation risky. Huynh (2019) summarised the use of smartphone devices in exotic animals. As with cats and dogs, smartphone apps can obtain ECGs in exotic animals, like penguins, though dolphin vocalisations can create artefacts. There are also smartphone attachments for direct ophthalmoscopy; the structures in the posterior segment could be identified in all animals, though focal light artefacts were common when the tapetum lucidum was photographed with certain devices. Non-clinically trained personnel were able to obtain acceptable images of the fundus, indicating potential for these devices in areas with limited resources. Otoscopes, laryngoscopes, cystoscopes, and endoscopes are available as smartphone attachments. Huynh (2019) states:

The main advantage for using a smartphone over a conventional endoscopic unit is the increased portability, accessibility, transmission, and lower price. Recording of the procedure and teletransmission can be performed instantaneously.

There are also point-of-care ultrasound devices that can be utilised for obtaining sonograms of exotic animals which may be particularly useful for focused assessment with sonography for trauma (FAST) exams (Huynh, 2019).

Vallone (2019) wrote about using smartphone technology in clinical ophthalmology without expensive accessory equipment. current smartphone technology, teleophthalmology is expected to increase (Vallone, 2019). Kanemaki et al. (2017) also used a smartphone and an indirect ophthalmoscopy lens to obtain various fundic images in dogs and cats. It was noted the photographer may need to adjust the exposure to account for the range of tones in the bright tapetum lucidum and the darker, pigmented non-tapetal area (Kanemaki et al., 2017).

Normal and abnormal brain functions include cognition, wakefulness, and sleep. These change during anaesthesia making it extremely difficult to determine the effects of trauma, disease, and epilepsy in animals. In human medicine, there have been advances with the use of electroencephalograms (EEG), but these are often difficult to obtain for animals. Cousillas et al. (2017) created an ambulatory EEG system that would allow horses to freely move about in their home location. The preliminary study was done with five horses trained 
for competition events. The study authors were able to obtain analysable EEGs from each horse and could identify alpha, beta, and gamma waves. The EEG system obtained its goal of being easily placed and fitted on each horse, the horse could be mobile in its home environment, and it did not put any more constraint on the animal than any usual gear. Further studies will need to be done to determine accuracy when asleep or under anaesthesia (Cousillas et al., 2017).

MacLarty et al. (2007) have compared a non-invasive biotelemetry shirt with whole body plethysmography in African green monkeys. The shirts provide a non-invasive method to continuously collect physiologic data, like respiration rate, ECG, activity, body temperature, blood pressure, EEG, and more without having to restrain the animal or surgically implant biotelemetric devices. The study concluded the biotelemetric shirt could be used in place of interpretive plethysmography studies and respiratory evaluation and allows for the animals to move about normally while continuously collecting data (MacLarty et al., 2007).

Diabetes mellitus in cats and dogs is a common endocrine problem that can be difficult to monitor and control. Home glucose monitoring is preferable to monitoring in the veterinary hospital because stress hyperglycaemia can lead to inaccurate results. Many clients are reluctant or unable to obtain blood samples from their pets for frequent blood glucose checks. A continuous blood glucose monitor can make this easier for cat owners, though the only one that does not require the pet owners to calibrate at home is the FreeStyle Libre (Abbott Laboratories, 2020). This sensor can be used to take multiple measurements for up to 14 days (Gottlieb \& Rand, 2018). After gathering the data from an at-home glucose curve, the client can schedule a virtual visit with the veterinarian and adjustments in the treatment plan can be made as needed. There are studies that have shown the accuracy of the FreeStyle Libre compared to glucose curves, and the clinical accuracy makes continuous glucose monitoring an attractive alternative for pet owners (Corradini et al., 2016; and Malerba et al., 2020).

High-tech wearables are tracking activity and providing clues to the early-onset of problems as well. Wearable devices can be attached to an animal's collar and track how active a dog is, how often a cat uses the litterbox, or if pets are making more frequent trips to the water bowl. Subtle changes may go unnoticed by the owner, but the data can reveal possible signs of infection, kidney disease, or an endocrinopathy. Food bowls can read an animal's microchip and be pre-programmed to open only for specific animals at set times to dispense a premeasured quantity of food. They also allow multi-pet homes to feed different foods and amounts to specific animals to assist with weight-loss plan or use of prescription foods. A report from Grand View Research states:

In coming years, the combination of wearable devices, mobile application, and data analytics technology can be a mainstream option for the value-based care. Some pet wearable companies are also providing cloud-based data analytics services along with their products, which can help veterinarians to diagnose and treat pets by providing valuable clinical information for real-time decision making (Jergler, 2018).

One of the major limitations to wearables is battery life, though it improves with each iteration (Jergler, 2018).

DISCUSSION

This literature review identified increasing literature about veterinary telemedicine including both peerreviewed studies and less rigorous articles in veterinary news magazines, like anecdotal reports, editorials, and opinion pieces. There is robust data to support virtual care for veterinary consults, as shown in a variety of studies, like those on radiology, pathology, cytology, behaviour, and cardiology. Data also supports 
teleconsulting to improve animal care in areas where resources may be limited, including rural areas, developing countries, and other remote locations. There is also a growing body of evidence to support $\mathrm{mHealth}$, the role and the use of wearable devices, and smartphone applications in veterinary medicine. Teleconsulting and mHealth have been shown to be effective across many species, including companion animals, exotics, equids, and food animals. There are fewer published studies about direct-to-consumer (DTC) telemedicine visits. These have been favourable, but most information about this has been gleaned from human health care. If human paediatrics is used as an analogy to veterinary medicine, studies in paediatric telehealth can lead to cautious optimism for veterinary medicine to observe similar outcomes. Veterinarians need to learn from paediatrician's successes and their deficits, for example judicious use of antimicrobials. Legal concerns will persist, though no published reports of veterinarians providing inadequate or substandard care via virtual care were identified. There was one lawsuit related to telemedicine, though that case involved the violation of VCPR laws, not the care actually provided (Ronald S. Hines, D.V.M., v. Texas State Board of Veterinary Medical Examiners, n.d.).

This review identified a number of published anecdotes of successful veterinary visits; however, those have not been analysed for publication in a peer-reviewed journal. The literature also lacks studies comparing in clinic visits with telehealth visits to determine if there is concordance between the findings of those exams. In general, the literature agrees telemedicine has a role in veterinary medicine. Based on studies of newlyboarded veterinary radiologists, it may also be important new graduates obtain experience in practice before performing virtual care visits unless an experienced veterinarian is available for support. It is important to note that telemedicine is a tool, and as such, it is not appropriate for every healthcare issue, situation, client, or animal. The challenges of providing veterinary care during the onset of COVID-19 and lockdowns in many areas led to a significant uptake in the utilisation of veterinary telehealth. This will lead to additional research and peer-reviewed publications that will further advance our knowledge of how telehealth is incorporated into veterinary practice, including benefits, risks, outcomes of those visits, including successes and adverse events. The creation of guidelines like the American Telemedicine Association and the Association of Paediatrics would most likely benefit those in the profession who would like to use veterinary virtual care in practice.

No one summed it up better than Bath (2006), in a letter to editor, who wrote, 'The conclusion is that Telemedicine is not something abstruse and only for the boffins. It is useful and simple to use, even today. It can only get better and its use become more widespread...' It is time to move forward with veterinary telemedicine using thoughtful consideration to the benefits and concerns that surround any tool integrated into veterinary practice.

\section{CONFLICT OF INTEREST}

This commissioned review was the basis for the 2019 ViVet Innovation Symposium talk, 'RCVS Knowledge Evidence into practice \& Initial findings of the telemedicine study' (Teller, 2019; and ViVet Press Office, 2020). The review was further expanded and updated for this paper.

Acknowledgements: The authors gratefully acknowledge L. M. Rey for assistance with data and reference management.

Funding: This review received no funding.

Competing interests: Moberly serves on the CABI Publishing North American Library Advisory Board and the VetStream Academic Advisory Board.

Teller serves on the air.Vet Advisory Board. 
1. Abbott Laboratories. (2020). Continuous Glucose Monitoring System. Available from: https://www.freestylelibre.us/ [Accessed 6 October 2020]

2. American Academy of Pediatrics. (2017). Operating Procedures for Pediatric Telehealth. Pediatrics, 140(2). DOI: https://doi.org/10.1542/peds.2017-1756

3. American College of Veterinary Behaviorists. (n.d.). Find a Board Certified Behaviorist. Available from: https://www.dacvb.org/search/custom.asp?id=4709 [Accessed 6 October 2020]

4. American Veterinary Medical Association. (2019). Principles of veterinary medical ethics of the AVMA. American Veterinary Medical Association. Available from: https://www.avma.org/policies/principlesveterinary-medical-ethics-avma [Accessed 6 October 2020]

5. American Veterinary Medical Association \& American Animal Hospital Association. (2018). The real-life rewards of virtual care: How to turn your hospital into a digitally connected practice with telehealth. American Animal Hospital Association. Available from: https://www.aaha.org/globalassets/05-pethealth-resources/virtual care.pdf [Accessed 6 October 2020]

6. American Veterinary Medical Association Practice Advisory Panel. (2017). Final report on telemedicine (p. 45). American Veterinary Medical Association. Available from: https://www.avma.org/sites/default/files/resources/Telemedicine-Report-2016.pdf [Accessed 6 October 2020]

7. Araújo, C. A. B. de, Francis, D. G. \& Almeida, L. a. M. (1999). The Internet as an educational resource for the Brazilian veterinarian. Veterinária Notícias, 5(2), 25-31.

8. Bath, G. F. (2006). Telemedicine in Africa. Journal of the South African Veterinary Association, 77(3), 107. DOI: https://doi.org/10.4102/jsava.v77i3.355

9. Bertram, C. A. \& Klopfleisch, R. (2017). The Pathologist 2.0: An Update on Digital Pathology in Veterinary Medicine. Veterinary Pathology, 54(5), 756-766.

DOI: https://doi.org/10.1177/0300985817709888

10. Bishop, G. T., Evans, B. A., Kyle, K. L. \& Kogan, L. R. (2018). Owner satisfaction with use of videoconferencing for recheck examinations following routine surgical sterilization in dogs. Journal of the American Veterinary Medical Association, 253(9), 1151-1157.

DOI: https://doi.org/10.2460/javma.253.9.1151

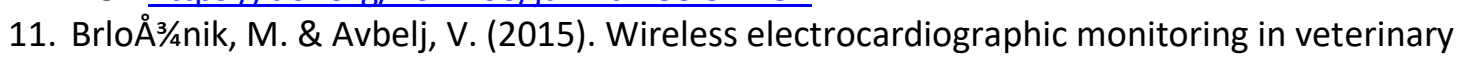
medicine. 2015 38th International Convention on Information and Communication Technology, Electronics and Microelectronics (MIPRO), 356-359. DOI: https://doi.org/10.1109/MIPRO.2015.7160294

12. Burke, B. L., Hall, R. W. \& Section on Telehealth Care. (2015). Telemedicine: Pediatric Applications. Pediatrics, 136(1), e293-308. DOI: https://doi.org/10.1542/peds.2015-1517

13. CAB Abstracts. (n.d.). Available from: http://www.ovid.com/product-details.31.html [Accessed 6 October 2020]

14. Carrozza, A. (2018). New Telemedicine App Connects Veterinarians with Behaviorists. Veterinarian's Money Digest. Available from: https://www.vmdtoday.com/news/new-telemedicine-app-connectsveterinarians-with-behaviorists [Accessed 6 October 2020]

15. Cary, M. \& Massecar, A. (2017). Veterinary Telehealth: What Is It, Where Are We, and What's Next? Today's Veterinary Practice. Available from: https://todaysveterinarypractice.com/insidenavcveterinary-telehealth-whats-next/ [Accessed 6 October 2020]

16. Clarivate Analytics. (n.d.). Web of Science. Available from: https://clarivate.com/webofsciencegroup/solutions/web-of-science/ [Accessed 6 October 2020]

17. Clinician's Brief. (n.d.). Available from: http://www.cliniciansbrief.com [Accessed 6 October 2020]

18. Corporation for Digital Scholarship. (n.d.). Zotero / Your personal research assistant. Available from: https://www.zotero.org/ [Accessed 6 October 2020] 
19. Corradini, S., Pilosio, B., Dondi, F., Linari, G., Testa, S., Brugnoli, F., Gianella, P., Pietra, M. \& Fracassi, F. (2016). Accuracy of a Flash Glucose Monitoring System in Diabetic Dogs. Journal of Veterinary Internal Medicine, 30(4), 983-988. DOI: https://doi.org/10.1111/jvim.14355

20. Cottam, N., Dodman, N. H., Moon-Fanelli, A. A. \& Patronek, G. J. (2008). Comparison of remote versus in-person behavioral consultation for treatment of canine separation anxiety. Journal of Applied Animal Welfare Science, 11(1), 28-41. DOI: https://doi.org/10.1080/10888700701729148

21. Cousillas, H., Oger, M., Rochais, C., Pettoello, C., Ménoret, M., Henry, S. \& Hausberger, M. (2017). An Ambulatory Electroencephalography System for Freely Moving Horses: An Innovating Approach. Frontiers in Veterinary Science, 4. DOI: https://doi.org/10.3389/fvets.2017.00057

22. Cushing, M. (2017). NAVC Keynote Commentary for the Veterinary Community: A Regulatory Road Map for Telehealth \& Pet Health Care. Today's Veterinary Practice. Available from: https://todaysveterinarypractice.com/navc-keynote-commentary-veterinary-communityregulatory-road-map-telehealth-pet-health-care/ [Accessed 6 October 2020]

23. Cushing, M. \& Lacroix, C. (2018). The state of the VCPR. Today's Veterinary Business. Available from: https://todaysveterinarybusiness.com/the-state-of-the-vcpr/ [Accessed 6 October 2020]

24. Devi, S., Singh, R. D., Ghasura, R. S., Sharma, M. K. \& Sharma, M. C. (2015). Telemedicine: A new rise of hope to animal health care sector-A Review. Agricultural Reviews, 36(2), 153. DOI: https://doi.org/10.5958/0976-0741.2015.00018.5

25. Dodman, N. H., Smith, A. \& Holmes, D. (2005). Comparison of the efficacy of remote consultations and personal consultations for the treatment of dogs which are aggressive towards their owners. Veterinary Record, 156(6), 168-170. DOI: https://doi.org/10.1136/vr.156.6.168

26. Donham, B. \& Wickett, M. L. (2018). Novel use of FaceTime video calling in a deployed setting to assist with the care of a military working dog. Canadian Journal of Surgery, 61(6 Suppl 1), S232-S234.

Available from: https://www.ncbi.nlm.nih.gov/pmc/articles/PMC6281462/ [Accessed 6 October 2020]

27. DVM 360. (n.d.). Available from https://www.dvm360.com/ [Accessed 6 October 2020]

28. Federation of Veterinarians of Europe. (2019). European Veterinary Code of Conduct, 2019 Edition. Available from: https://www.fve.org/cms/wpcontent/uploads/FVE Code of Conduct 2019 R1 WEB.pdf [Accessed 6 October 2020]

29. Fischetti, A. J., Shiroma, J. T. \& Poteet, B. A. (2017). Academic and private practice partnerships in veterinary radiology residency training. Veterinary Radiology \& Ultrasound: The Official Journal of the American College of Veterinary Radiology and the International Veterinary Radiology Association, 58(4), 367-372. DOI: https://doi.org/10.1111/vru.12507

30. Flemming, D. D. (2003). Practicing veterinary medicine across state lines-Are you legal? Journal of the American Veterinary Medical Association, 222(1), 30-33.

DOI: https://doi.org/10.2460/javma.2003.222.30

31. Forlani, E., De Lazzari, C., Maiolino, P., Poli, A., Pugliese, A., Rabbito, C. \& Sicurello, F. (2010). The first veterinary telemedicine study group. Journal of Telemedicine and Telecare, 16(3), 162-163.

DOI: https://doi.org/10.1258/itt.2009.009006

32. Freiman, S. (2019, May 3). Telemedicine from the frontline: A practicing veterinarian's perspective. Veterinary Practice News. Available from: https://www.veterinarypracticenews.com/telemedicine-from-the-frontline-a-practicingveterinarians-perspective/ [Accessed 6 October 2020]

33. Google. (n.d.). Google Scholar. Available from: https://scholar.google.com/ [Accessed 6 October 2020]

34. Gottlieb, S. \& Rand, J. (2018). Managing feline diabetes: Current perspectives. Veterinary Medicineâ€ $€^{-}$: Research and Reports, 9, 33-42. DOI: https://doi.org/10.2147/VMRR.S125619

35. Gyles, C. (2019). Veterinary telemedicine. The Canadian Veterinary Journal = La Revue Veterinaire Canadienne, 60(2), 119-122. Available

from: https://www.ncbi.nlm.nih.gov/pmc/articles/PMC6340261/ [Accessed 6 October 2020] 
36. Hawk, C. (2018). Veterinary Telemedicine Perception and Utilization Intention [Honors thesis, University of Tennessee Knoxville]. Available

from: https://trace.tennessee.edu/cgi/viewcontent.cgi?article=3266\&context=utk chanhonoproj [Acc essed 6 October 2020]

37. Hebert, P., Latouche, J. S., Menard, M. \& Papageorges, M. (2001). Telecytology. Clinical Techniques in Small Animal Practice, 16(2), 122-124. DOI: https://doi.org/10.1053/svms.2001.26235

38. Hess, L. (2017). Telemedicine: The Future of Veterinary Practice. Journal of Avian Medicine and Surgery, 31(2), 165-171. DOI: https://doi.org/10.1647/1082-6742-31.2.165

39. Huynh, M. (2019). Smartphone-Based Device in Exotic Pet Medicine. The Veterinary Clinics of North America. Exotic Animal Practice, 22(3), 349-366. DOI: https://doi.org/10.1016/j.cvex.2019.05.001

40. Ilukor, J., Nielsen, T. \& Birner, R. (2014). Determinants of referrals from paraprofessionals to veterinarians in Uganda and Kenya. Preventive Veterinary Medicine, 114(3-4), 164-173.

DOI: https://doi.org/10.1016/j.prevetmed.2014.02.009

41. Ishmael, S. (2015, March 3). Exploring new frontiers with veterinary telemedicine. Beef Magazine. Available from: https://www.beefmagazine.com/beef-vet/exploring-new-frontiers-veterinarytelemedicine [Accessed 6 October 2020]

42. Jack, D. C. (1999). The legal implications of veterinary telemedicine and telecare. Journal of Telemedicine and Telecare, 5(Suppl 1), S80-84. DOI: https://doi.org/10.1258/1357633991932685

43. Jergler, D. (2018). High-tech remote health monitoring. Veterinary Practice News. Available from: https://www.veterinarypracticenews.com/high-tech-remote-health-monitoring/ [Accessed 6 October 2020]

44. Kane, C. K. \& Gillis, K. (2018). The Use Of Telemedicine By Physicians: Still The Exception Rather Than The Rule. Health Affairs (Project Hope), 37(12), 1923-1930.

DOI: https://doi.org/10.1377/hlthaff.2018.05077

45. Kanemaki, N., Inaniwa, M., Terakado, K., Kawarai, S. \& Ichikawa, Y. (2017). Fundus photography with a smartphone in indirect ophthalmoscopy in dogs and cats. Veterinary Ophthalmology, 20(3), 280-284. DOI: https://doi.org/10.1111/vop.12399

46. Kogan, L. R., Hellyer, P. W., Ruch-Gallie, R., Rishniw, M. \& Schoenfeld-Tacher, R. (2016). Veterinarians' Use and Perceptions of Information and Communication Technologies. Medical Research Archives, 4(2), Article 2. Available from: https://journals.ke-i.org/mra/article/view/502 [Accessed 6 October 2020]

47. Kogan, L., Schoenfeld, R. \& Santi, S. (2019). Medical Updates and Appointment Confirmations: Pet Owners' Perceptions of Current Practices and Preferences. Frontiers in Veterinary Science, 6. DOI: https://doi.org/10.3389/fvets.2019.00080

48. Kraus, M. S., Gelzer, A. R. \& Rishniw, M. (2016). Detection of heart rate and rhythm with a smartphone-based electrocardiograph versus a reference standard electrocardiograph in dogs and cats. Journal of the American Veterinary Medical Association, 249(2), 189-194.

DOI: https://doi.org/10.2460/javma.249.2.189

49. Lacroix, C. (2017). Telemedicine: From your exam room to their living room. Veterinary Technician, Practice Management, PM94-PM97.

50. Lanevschi-Pietersma, A., Boroffka, S., Pereira, Y. M., Penzo, C., Pizzi, R., Rudorf, H. \& Tartarelli, C. (2011). Telemedicine: A time management and learning tool for vets and service clinics and what it can offer to pet owners. European Journal of Companion Animal Practice, 21(1), 73-76. Available from: https://www.zora.uzh.ch/id/eprint/123884/1/ejcap 21 1.pdf\#page=67 [Accessed 6 October 2020]

51. Lichtenberger, J., Meurs, K. M. \& Côté, E. (2018). Preliminary Assessment of a Novel 14-Day Electrocardiographic Adhesive Patch Monitor in Dogs. Journal of the American Animal Hospital Association, 54(3), 138-143. DOI: https://doi.org/10.5326/JAAHA-MS-6626

52. Limb, M. (2018). Telemedicine: Are we nearly there yet? The Veterinary Record, 182(20), 564-565. DOI: https://doi.org/10.1136/vr.k2187 
53. MacLarty, A., Nalca, A. \& Roy, C. (2007). Comparison of non-invasive bio-telemetry shirt system to whole body plethysmography in African Green Monkeys (Chlorocebus aethiops). Online Journal of Veterinary Research, 11(1), 1-9. Available

from: http://onljvetres.com/telemetryabs2006.htm [Accessed 6 October 2020]

54. Magalhães-Sant'Ana, M., Conceição Peleteiro, M. \& Stilwell, G. (2020). Opinions of Portuguese

Veterinarians on Telemedicine-A Policy Delphi Study. Frontiers in Veterinary Science, 7.

DOI: https://doi.org/10.3389/fvets.2020.00549

55. Mahendran, S. A., Booth, R., Bell, N. J. \& Burge, M. (2017). Randomised positive control trial of NSAID and antimicrobial treatment for calf fever caused by pneumonia. The Veterinary Record, 181(2), 45.

DOI: https://doi.org/10.1136/vr.104057

56. Maiolino, P., Restucci, B., Papparella, S. \& De Vico, G. (2006). Evaluation of static telepathology in veterinary diagnostic cytology. Veterinary Clinical Pathology, 35(3), 303-306.

DOI: https://doi.org/10.1111/j.1939-165x.2006.tb00135.x

57. Malerba, E., Cattani, C., Baldo, F. D., Carotenuto, G., Corradini, S., Golinelli, S., Drudi, I., \& Fracassi, F. (2020). Accuracy of a flash glucose monitoring system in dogs with diabetic ketoacidosis. Journal of Veterinary Internal Medicine, 34(1), 83-91. DOI: https://doi.org/10.1111/jvim.15657

58. Mang, A. V., Buczinski, S., Booker, C. W. \& Timsit, E. (2015). Evaluation of a Computerâ€?laided Lung Auscultation System for Diagnosis of Bovine Respiratory Disease in Feedlot Cattle. Journal of Veterinary Internal Medicine, 29(4), 1112-1116. DOI: https://doi.org/10.1111/jvim.12657

59. Marr, C. M. (2001). Advances in Equine Ultrasonography. Veterinary Clinics of North America: Equine Practice, 17(2), 305-317. DOI: https://doi.org/10.1016/S0749-0739(17)30064-0

60. Mars, M. \& Auer, R. E. J. (2006). Telemedicine in veterinary practice. Journal of the South African Veterinary Association, 77(2), 75-78. DOI: https://doi.org/10.4102/isava.v77i2.348

61. Marshall, K. (2019). Ethical issues for today's veterinarian in the digital age. Veterinary Practice News. Available from: https://www.veterinarypracticenews.com/ethical-issues-for-todays-veterinarian-inthe-digital-age/ [Accessed 6 October 2020]

62. Martinelli, M. (2001). Digital Imaging Advances and the Future. Veterinary Clinics of North America: Equine Practice, 17(2), 275-295. DOI: https://doi.org/10.1016/S0749-0739(17)30062-7

63. Mazan, M. R., Kay, G., Merriam, J. G., Bubeck, K. \& Jenei, T. (2015). Patients without borders: Collaborative telemedicine among university, private, and pro bono practices. Proceedings of the 61st Annual Convention of the American Association of Equine Practitioners, Las Vegas, Nevada, USA, December 5-9, 2015, 323-325.

64. McCorkell, R., Wynne-Edwards, K., Windeyer, C., Schaefer, A. \& UCVM Class of 2013. (2014). Limited efficacy of Fever $\operatorname{Tag}\left({ }^{\circledR}\right)$ temperature sensing ear tags in calves with naturally occurring bovine respiratory disease or induced bovine viral diarrhea virus infection. The Canadian Veterinary Journal = La Revue Veterinaire Canadienne, 55(7), 688-690. Available from: https://www.ncbi.nlm.nih.gov/pmc/articles/PMC4060915/ [Accessed 6 October 2020]

65. McSwain, S. (2019). Clarification for veterinary medicine [Personal communication].

66. McSwain, S. D., Bernard, J., Burke, B. L., Cole, S. L., Dharmar, M., Hall-Barrow, J., Herendeen, N., Herendeen, P., Krupinski, E. A., Martin, A., McCafferty, D., Mulligan, D. A., North, S., Ruschman, J., Waller, M., Webster, K., Williams, S., Yamamoto, S. \& Yeager, B. (2017). American Telemedicine Association Operating Procedures for Pediatric Telehealth. Telemedicine Journal and E-Health: The Official Journal of the American Telemedicine Association, 23(9), 699-706.

DOI: https://doi.org/10.1089/tmj.2017.0176

67. Milani, M. (2009). High-tech client communication. The Canadian Veterinary Journal = La Revue Veterinaire Canadienne, 50(12), 1291-1294. Available from: https://www.ncbi.nlm.nih.gov/pmc/articles/PMC2777297/ [Accessed 6 October 2020]

68. National Library of Medicine, National Center for Biotechnology Information \& National Institutes of Health. (n.d.). Home-PubMed-NCBI (Legacy) [Computer software]. Available from: https://www.ncbi.nlm.nih.gov/pubmed/ [Accessed 6 October 2020] 
69. National Research Council. (2013). Workforce Needs in Veterinary Medicine. The National Academies Press. DOI: https://doi.org/10.17226/13413

70. Navas de Solis, C., Bevevino, K., Doering, A., O’Gan, D., Teller, L. \& Underwood, C. (2019). Real-time telehealth using ultrasonography is feasible in equine practice. Equine Veterinary Education. DOI: https://doi.org/10.1111/eve.13177

71. Nelson-Pratt, A. (2018). Telemedicine debate is taking far too long. Veterinary Record, 183(21), 663663. DOI: https://doi.org/10.1136/vr.k5073

72. Niessen, S. \& Forcada, Y. (2019, March 11). Telemedicine advances: Putting specialist help in all practices. Vet Times. Available from: https://www.vettimes.co.uk/article/telemedicine-advancesputting-specialist-help-in-all-practices/ [Accessed 6 October 2020]

73. Noel, P. G., Fischetti, A. J., Moore, G. E. \& Le Roux, A. B. (2016). Off-site smartphone vs. Standard workstation in the radiographic diagnosis of small intestinal mechanical obstruction in dogs and cats. Veterinary Radiology \& Ultrasound: The Official Journal of the American College of Veterinary Radiology and the International Veterinary Radiology Association, 57(5), 457-461.

DOI: https://doi.org/10.1111/vru.12383

74. Noffsinger, T., Brattain, K., Quakenbush, G. \& Taylor, G. (2014). Field results from Whisper ${ }^{\circledR}$ stethoscope studies. Animal Health Research Reviews, 15(2), 142-144.

DOI: https://doi.org/10.1017/S1466252314000218

75. Palmer, S. (n.d.). ProQuest Platform: About. Available from: https://proquest.libguides.com/proquestplatform/about [Accessed 6 October 2020]

76. Papageorges, M. (2001). Veterinary telemedicine. Introduction. Clinical Techniques in Small Animal Practice, 16(2), 87-89. DOI: https://doi.org/10.1053/svms.2001.26224

77. Papageorges, M., Hanson, J. \& Girard, E. (1998). Telesonography may solve ultrasonography dilemma. Journal of the American Veterinary Medical Association, 212(7), 948.

78. Papageorges, M., Hanson, J., Girard, E., Leveille, R., Hebert, P., Latouche, J. S. \& Menard, M. (2001). How to make telemedicine work. Clinical Techniques in Small Animal Practice, 16(2), 111-114. DOI: https://doi.org/10.1053/svms.2001.26230

79. Papageorges, M. \& Hebert, P. (2001a). Choosing your telemedicine system. Clinical Techniques in Small Animal Practice, 16(2), 107-110. DOI: https://doi.org/10.1053/svms.2001.26228

80. Papageorges, M. \& Hebert, P. (2001b). Other telemedicine applications. Clinical Techniques in Small Animal Practice, 16(2), 125-126. DOI: https://doi.org/10.1053/svms.2001.26236

81. Papageorges, M. \& Hebert, P. (2001c). Telemedicine using standard Internet technologies (telemedicine for less than $\$ 1,000)$. Clinical Techniques in Small Animal Practice, 16(2), 102-106. DOI: https://doi.org/10.1053/svms.2001.26227

82. Papageorges, M, \& Tilley, L. (2001). Why telemedicine? Clinical Techniques in Small Animal Practice, 16(2), 90-94. DOI: https://doi.org/10.1053/svms.2001.26225

83. Papageorges, M. (Ed.). (2001). Clinical Techniques in Small Animal Practice: Veterinary Telemedicine: Vol. 16:2. Available from: https://www.sciencedirect.com/journal/clinical-techniques-in-small-animalpractice/vol/16/issue/2 [Accessed 6 October 2020]

84. Penzo, C. \& Pietersma, A. (2012). Teleoncology: A tool for supporting cancer care. In Practice, 34(10), 608-612. DOI: https://doi.org/10.1136/inp.e6321

85. Poteet, B. A. (2008). Veterinary teleradiology. Veterinary Radiology \& Ultrasound, 49(s1), S33-S36. DOI: https://doi.org/10.1111/j.1740-8261.2007.00331.x

86. ProQuest. (n.d.). ProQuest-Dissertations \& Theses. Available from: http://www.proquest.com/libraries/academic/dissertations-theses [Accessed 6 October 2020]

87. Quimby, J. M., Olea-Popelka, F. \& Lappin, M. R. (2009). Comparison of digital rectal and microchip transponder thermometry in cats. Journal of the American Association for Laboratory Animal Science: JAALAS, 48(4), 402-404. Available

from: https://www.ingentaconnect.com/contentone/aalas/jaalas/2009/00000048/00000004/art0001 $\underline{1}$ [Accessed 6 October 2020] 
88. Ray, K. N., Shi, Z., Gidengil, C. A., Poon, S. J., Uscher-Pines, L. \& Mehrotra, A. (2019). Antibiotic Prescribing During Pediatric Direct-to-Consumer Telemedicine Visits. Pediatrics, 143(5).

DOI: https://doi.org/10.1542/peds.2018-2491

89. Rethlefsen, M., Ayala, A. P., Kirtley, S., Koffel, J. \& Waffenschmidt, S. (2019). PRISMA-S Draft. DOI: https://doi.org/10.17605/OSF.IO/7NCYS

90. Robben, J. H., Melsen, D. N., Almalik, O., Roomer, W. \& Endenburg, N. (2016). Evaluation of a virtual pet visit system with live video streaming of patient images over the Internet in a companion animal intensive care unit in the Netherlands. Journal of Veterinary Emergency and Critical Care (San Antonio, Tex.: 2001), 26(3), 384-392. DOI: https://doi.org/10.1111/vec.12458

91. Robertson, T. A. (1999). Telemedicine-Creating the virtual veterinary hospital. Compendium on Continuing Education for the Practicing Veterinarian, 21(2), 128-133.

92. Ronalds Hines, D.V.M., Plaintiff, v. Jessica Quillivan, D.V.M., in her official capacity as President of the Texas State Board of Veterinary Medical Examiners; KEITH PARDUE, in his official capacity as Vice President of the Texas State Board of Veterinary Medical Examiners; SANDRA“LYNN" CRINER, D.V.M., in her official capacity as Secretary of the Texas State Board of Veterinary Medical Examiners; MICHAELWHITE, D.V.M., in his official capacity as a Member of the Texas State Board of Veterinary Medical Examiners; SAMANTHA MIXON,D.V.M., in her official capacity as a Member of the Texas State Board of Veterinary Medical Examiners; RANDALL SKAGGS, D.V.M., in his official capacity as a Member of the Texas State Board of Veterinary Medical Examiners; CARLOS CHACON, in his official capacity as a Member of the Texas State Board of Veterinary Medical Examiners; SUE ALLEN,L.V.T., in her official capacity as a Member of the Texas State Board of Veterinary Medical Examiners; and GEORGE ANTUNA, in his official capacity as a Member of the Texas State Board of Veterinary Medical Examiners, Defendants, Case 1:18-cv-155 (United States District Court for the Southern District of Texas, Brownsville Division). Available from: https://ij.org/wp-content/uploads/2018/10/Texas-FreeSpeech-Case-Complaint.pdf [Accessed 6 October 2020]

93. Rose, R. (2017). Empowering your veterinary staff to step outside the box. Large Animal, Practice Management, PM151-PM152.

94. Royal College of Veterinary Surgeons. (n.d.). Code of Professional Conduct for Veterinary SurgeonsProfessionals. Royal College of Veterinary Surgeons. Available from: https://www.rcvs.org.uk/settingstandards/advice-and-guidance/code-of-professional-conduct-for-veterinary-surgeons/ [Accessed 6 October 2020]

95. Royal College of Veterinary Surgeons. (2018). RCVS review of the use of telemedicine within veterinary practice: Summary analysis. Royal College of Veterinary Surgeons. Available from: https://www.rcvs.org.uk/document-library/telemedicine-consultation-summary/ [Accessed 6 October 2020]

96. Shigekawa, E., Fix, M., Corbett, G., Roby, D. H. \& Coffman, J. (2018). The Current State Of Telehealth Evidence: A Rapid Review. Health Affairs (Project Hope), 37(12), 1975-1982.

DOI: https://doi.org/10.1377/hlthaff.2018.05132

97. Siew, L., Hsiao, A., McCarthy, P., Agarwal, A., Lee, E. \& Chen, L. (2016). Reliability of Telemedicine in the Assessment of Seriously III Children. Pediatrics, 137(3). DOI: https://doi.org/10.1542/peds.2015$\underline{0712}$

98. Teller, L. (2019). RCVS Knowledge - Evidence into practice \& Initial findings of the telemedicine study. Available from: https://www.youtube.com/watch?v=epiQinmmEKw\&feature=youtu.be [Accessed 12 October 2020]

99. Tilley, L. P., Miller, M. S., Wortman, J. A., Biery, D. N. \& Coward, B. H. (1986). Teleradiology in veterinary medicine. Seminars in Veterinary Medicine and Surgery (Small Animal), 1(2), 172-175.

100. Today's Veterinary Business / An Official Journal of the NAVC. (n.d.). Today's Veterinary Business. Available from: https://todaysveterinarybusiness.com/ [Accessed 6 October 2020]

101. Vallone, L. V. (2019). Smartphone Technology in Clinical Ophthalmology. Available from: http://www.cliniciansbrief.com/article/smartphone-technology-clinicalophthalmology [Accessed 6 October 2020] 
102. Veterinary Team Brief / Clinician's Brief. (n.d.). Available from: https://www.cliniciansbrief.com/veterinary-team-brief [Accessed 6 October 2020]

103. Vezzosi, T., Buralli, C., Marchesotti, F., Porporato, F., Tognetti, R., Zini, E. \& Domenech, O. (2016). Diagnostic accuracy of a smartphone electrocardiograph in dogs: Comparison with standard 6-lead electrocardiography. Veterinary Journal (London, England: 1997), 216, 33-37. DOI: https://doi.org/10.1016/j.tvjl.2016.06.013

104. Vezzosi, Tommaso, Sgorbini, M., Bonelli, F., Buralli, C., Pillotti, M., Meucci, V. \& Tognetti, R. (2018). Evaluation of a Smartphone Electrocardiograph in Healthy Horses: Comparison With Standard Baseapex Electrocardiography. Journal of Equine Veterinary Science, 67, 61-65.

DOI: https://doi.org/10.1016/j.jevs.2018.03.006

105. ViVet Press Office. (2020). Symposium 2019 reports: Chris Gush and Lori Teller. Available from: https://www.vivet.org.uk/chris-gush-and-lori-teller-rcvs-knowledge/ [Accessed 12 October 2020]

106. Warren, S., Martinez, A., Sobering, T. \& Andresen, D. (2008). Electrocardiographic pill for cattle heart rate determination. Conference Proceedings: ... Annual International Conference of the IEEE Engineering in Medicine and Biology Society. IEEE Engineering in Medicine and Biology Society. Annual Conference, 2008, 4852-4855. DOI: https://doi.org/10.1109/IEMBS.2008.4650300

107. World Organisation for Animal Health (OIE). (2019). Glossary. In Terrestrial Animal Health Code (Vol. 12). World Organisation for Animal Health (OIE). Available from: https://www.oie.int/index.php?id=169\&L=0\&htmfile=glossaire.htm [Accessed 6 October 2020] 


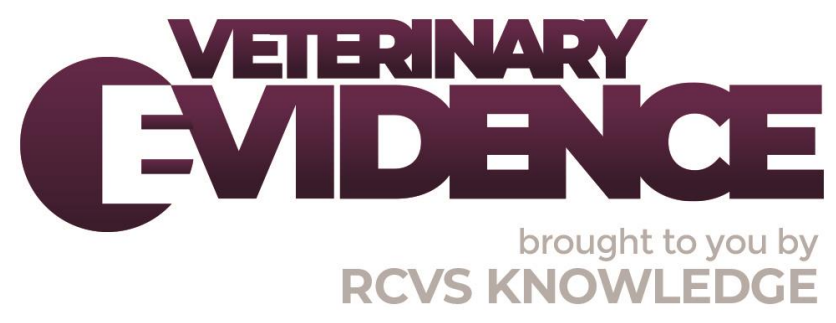

\section{Intellectual Property Rights}

Authors of Knowledge Summaries submitted to RCVS Knowledge for publication will retain copyright in their work, and will be required to grant RCVS Knowledge a non-exclusive license of the rights of copyright in the materials including but not limited to the right to publish, re-

publish, transmit, sell, distribute and otherwise use the materials in all languages and all media throughout the world, and to license or permit others to do so.

\section{Disclaimer}

Any opinions expressed in articles and other publication types published in Veterinary Evidence are the author's own and do not necessarily reflect the view of the RCVS Knowledge. Veterinary Evidence is a resource to help inform, and the content herein should not override the responsibility of the practitioner. Practitioners should also consider factors such as individual clinical expertise and judgement along with patient's circumstances and owners' values. Authors are responsible for the accuracy of the content. While the Editor and Publisher believe that all content herein are in accord with current recommendations and practice at the time of publication, they accept no legal responsibility for any errors or omissions, and make no warranty, express or implied, with respect to material contained within.

For further information please refer to our Terms of Use.

RCVS Knowledge is the independent charity associated with the Royal College of Veterinary Surgeons (RCVS). Our ambition is to become a global intermediary for evidence based veterinary knowledge by providing access to information that is of immediate value to practicing veterinary professionals and directly contributes to evidence based clinical decision-making.

https://www.veterinaryevidence.org/

RCVS Knowledge is a registered Charity No. 230886.

Registered as a Company limited by guarantee in England and Wales No. 598443.

Registered Office: Belgravia House, 62-64 Horseferry Road, London SW1P 2AF

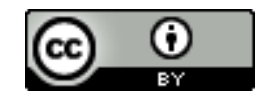

This work is licensed under a Creative Commons Attribution 4.0 International License. 